Received: 22 June 2017

Accepted: 27 September 2017

Published online: 16 October 2017

\section{Conversion of FeCo from soft to hard magnetic material by lattice engineering and nanopatterning}

Takashi Hasegawa, Shunsuke Kanatani, Miyu Kazaana, Kairi Takahashi, Kohei Kumagai, Maiko Hirao \& Shunji Ishio

The development of magnetic materials with large uniaxial magnetic anisotropy $\left(K_{\mathrm{u}}\right)$ and high saturation magnetization has attracted much attention in various areas such as high-density magnetic storage, spintronic devices, and permanent magnets. Although FeCo alloys with the body-centred cubic structure exhibit the highest $M_{\mathrm{s}}$ among all transition metal alloys, their low $K_{\mathrm{u}}$ and coercivity $\left(H_{\mathrm{c}}\right)$ make them unsuitable for these applications. However, recent first-principles calculations have predicted large $K_{\mathrm{u}}$ for the FeCo films with the body-centred tetragonal structure. In this work, we experimentally investigated the hard magnetic properties and magnetic domain structures of nanopatterned FeCo alloy thin films. As a result, a relatively large value of the perpendicular uniaxial magnetic anisotropy $K_{\mathrm{u}}=2.1 \times 10^{6} \mathrm{~J} \cdot \mathrm{m}^{-3}$ was obtained, while the $H_{\mathrm{c}}$ of the nanopatterned FeCo layers increased with decreasing dot pattern size. The maximum $H_{\mathrm{c}}$ measured in this study was $4.8 \times 10^{5} \mathrm{~A} \cdot \mathrm{m}^{-1}$, and the corresponding value of $\mu_{0} H_{c}$ was $0.60 \mathrm{~T}$, where $\mu_{0}$ represented the vacuum permeability.

The continuously increasing power consumption in motors $^{1}$ and data storage devices ${ }^{2,3}$ containing permanent magnets has become a serious issue. In this regard, enhancing the performance of permanent magnets represents the simplest and most efficient method for reducing their power consumption. Since the energy utilized by permanent magnets depends on two parameters, coercivity $\left(H_{\mathrm{c}}\right)$ and saturation magnetization $\left(M_{\mathrm{s}}\right)$, high-performance motors must possess relatively high values of $H_{\mathrm{c}}$ and $M_{\mathrm{s}}$ to achieve sufficiently high flux densities. The ideal maximum energy product $(B H)_{\max }$ is defined by the following formula:

$$
(B H)_{\max }=M_{\mathrm{s}}^{2} /\left(4 \mu_{0}\right)
$$

where $\mu_{0}$ is the vacuum permeability. This expression is based on the single domain theory that assumes hard magnetic properties of a material with a sufficiently high value of $H_{\mathrm{c}}^{4}$. Among commercial permanent magnets ${ }^{1}$, FeNdB exhibits the highest $(B H)_{\max }$ of around $500 \mathrm{~kJ} \cdot \mathrm{m}^{-3}$, while relatively high uniaxial magnetic anisotropy $\left(K_{\mathrm{u}}\right)$ is required for high-density magnetic storage, as illustrated by various bit-patterned media ${ }^{2}$. In order to prevent the generation of thermal fluctuations and, therefore, bit errors, the thermal stability factor $K_{\mathrm{u}} V$ of a magnetic layer must be much higher than the thermal energy $k_{\mathrm{B}} T$ (here $V$ is the volume of a magnetic dot, $k_{\mathrm{B}}$ is the Boltzmann constant, and $T$ is the temperature). The magnitude of $V$ decreases with an increase in the storage density; hence, a relatively high $K_{\mathrm{u}}$ is required for maintaining high $K_{\mathrm{u}} V$. A high $M_{\mathrm{s}}$ is also required for reading the magnetic flux from the recorded bits with low noise. By increasing the storage density, the number of hard disk drives utilized in data centres can be decreased, thereby reducing their power consumption.

Soft and hard magnetic materials are characterized by low and high $H_{\mathrm{c}}$ values, respectively. FeCo with the body-centred cubic (bcc) structure is a known soft magnetic material with the highest $M_{\mathrm{s}}$ among the currently studied transition metal alloys ${ }^{5,6}$. Although FeCo alloys exhibit relatively high $M_{\mathrm{s}}$, their low values of $K_{\mathrm{u}}$ and $H_{\mathrm{c}}$ make them unsuitable for the application as permanent magnets in motors and data storage. However, recent first-principles calculations have predicted a relatively high $K_{\mathrm{u}}$ for FeCo with the body-centred tetragonal (bct) structure $^{7-11}$. The experimental $K_{\mathrm{u}}$ values have been also obtained for FeCo alloys with the bct structure ${ }^{12-22}$; they included a large $K_{\mathrm{u}}$ of over $10^{6} \mathrm{~J} \cdot \mathrm{m}^{-3}$ and lattice parameter ratio $(c / a)$ between 1.15 and $1.25^{22}$. In addition, the $M_{\mathrm{s}}$ values that are equal to $85 \%$ of those measured for the bcc bulk $\mathrm{Fe}_{50} \mathrm{Co}_{50}$ have been reported as well ${ }^{7,22}$. However, the experimentally obtained $H_{\mathrm{c}}$ values were below $1 \%\left(10^{3}\right.$ to $\left.10^{4} \mathrm{~A} \cdot \mathrm{m}^{-112-22}\right)$ of the theoretical value. 
The fabrication of dot patterns not only helps achieve high coercivity, determine a single domain size, and investigate the magnetization reversal process ${ }^{21-25}$; it is also essential for the development of bit-patterned media and innovative permanent magnets. A theoretical $H_{c}$ value can be obtained when the dot volume is smaller than the size of a single domain after the coherent magnetization rotation according to the following expression derived from the single domain theory ${ }^{4}$ :

$$
H_{\mathrm{c}}=2 K_{\mathrm{u}} / M_{\mathrm{s}}
$$

The $H_{c}$ of dot patterns depends on the dot size and approaches the theoretical value in multilayer systems ${ }^{23}$. However, its magnitude for the ordered alloys is much lower than the theoretical value because of the non-uniformity of $K_{\mathrm{u}}$ and other physical parameters related to the non-uniformity of the order parameter inside the dots resulting from the annealing and ion milling processes ${ }^{24}$. The non-uniformity of the produced dot pattern can provide nucleation sites that induce magnetization reversal at a lower magnetic field than the theoretical $H_{\mathrm{c}}$ value. In that case, the demagnetizing field $\left(H_{\mathrm{d}}\right)$ must be taken into account to obtain a high $H_{\mathrm{c}}$ value. The magnitude of $H_{\mathrm{d}}$ is defined by the following expression:

$$
H_{\mathrm{d}}=-N M_{\mathrm{s}} / \mu_{0}
$$

where $\mathrm{N}$ is the demagnetizing factor, which depends only on the shape of the magnetic material ${ }^{4}$. The demagnetizing factors for the oblate spheroid having a major axis $(D, D, t)$ with $k=D / t>1$ are equal to

$$
\begin{gathered}
N_{\mathrm{D}}=1 /\left(2\left(k^{2}-1\right)\right)\left(\left(k^{2} \arccos (1 / k)-1\right) / \sqrt{k^{2}-1}\right) \\
N_{\mathrm{t}}=1-2 N_{\mathrm{D}} .
\end{gathered}
$$

For a discoidal dot, the parameters $D$ and $t$ correspond to its diameter and thickness, respectively. A larger $t$ results in a lower $H_{\mathrm{d}}$, which in turn produces a higher $H_{\mathrm{c}}$ according to the following expression:

$$
H_{\mathrm{c}}=p\left(2 K_{\mathrm{u}} / M_{\mathrm{s}}\right)-H_{\mathrm{d}}
$$

where $\mathrm{p}$ is the factor related to the nucleation sites, which describes the non-uniformity of the physical parameters inside the dots $(p<1)$.

In this study, nanopatterned bct FeCo alloy layers were fabricated, and the effects of the dot size and FeCo layer thickness on their magnetic domain structure and $H_{c}$ value, respectively, were investigated.

Introduction of uniaxial magnetocrystalline anisotropy by lattice engineering. Prior to nanopatterning, the crystal structure and magnetic properties of $\mathrm{FeCo} / \mathrm{Rh} / \mathrm{MgO}$ (substrate) continuous films were evaluated. Accordingly, 10 at.\% $\mathrm{Al}$ was added to the $\mathrm{FeCo}$ alloy, and the resulting ternary alloy is hereafter referred to as $\mathrm{FeCo}(\mathrm{Al}) . \mathrm{FeCo}(\mathrm{Al})$ forms a $\mathrm{CsCl}$-type (B2) ordered phase over a wide composition range ${ }^{26}$; hence, $K_{\mathrm{u}}$ was expected to be enhanced through $B 2$ ordering and strong magnetoelastic interactions. Previous experiments have shown that the addition of 10 at.\% $\mathrm{Al}$ results in the maximum value of $K_{\mathrm{u}}^{27}$. The experimental composition of the alloy determined by electron probe X-ray microanalysis can be described by the formula $\left(\mathrm{Fe}_{0.5} \mathrm{Co}_{0.5}\right)_{90} \mathrm{Al}_{10}$. The thickness of the $\mathrm{Rh}$ layer was fixed at $20.0 \mathrm{~nm}$, while that of the $\mathrm{FeCo}(\mathrm{Al})$ layer $t$ was varied between 2.0 and $20.0 \mathrm{~nm}$. Rh was selected as a buffer layer material because it exhibited the lattice mismatch $\left(a_{\mathrm{FeCo}}-a_{\mathrm{Rh}} / \sqrt{2}\right) / a_{\mathrm{FeCo}} \approx 0.05$, which was suitable for the introduction of epitaxial distortion into the $\mathrm{FeCo}(\mathrm{Al})$ structure. The [001] growth of FeCo on the Rh buffer surface characterized by the structural relation bct FeCo $(001)[110] / / \mathrm{Rh}(001)[100] / / \mathrm{MgO}(001)[100]$ was confirmed by scanning transmission electron microscopy ${ }^{22}$. The order parameter estimated via X-ray diffraction (XRD) with synchrotron radiation was equal to 0.1-0.2.

The lattice parameter ratio $c / a$ estimated for the bct unit cell of a FeCo(Al) layer (where $c$ and $a$ were evaluated via out-of-plane and in-plane XRD measurements) is plotted as a function of the film thickness $(t)$ in Fig. 1a. The inset illustrates the relationship between the face-centred cubic (fcc) and bct crystallographic unit cells obtained at $c / a=\sqrt{2} \cong 1.41$. The $c / a$ value at $t=0$ is equal to 1.41 , which is consistent with the value determined for the $\mathrm{Rh}$ fcc structure. The $c / a$ value decreased with increasing $t$, reaching 1.01 at $t=20.0 \mathrm{~nm}$ because of the structural relaxation, and was slightly larger than that required for a bcc structure.

Figure $1 \mathrm{~b}$ shows the perpendicular magnetization curves $(\perp)$ obtained using the magneto-optic Kerr effect (MOKE) method, while Fig. $1 \mathrm{c}$ displays the $K_{\mathrm{u}}$ values plotted as a function of $t$. The $K_{\mathrm{u}}$ values were calculated via the following equation:

$$
K_{\mathrm{u}}=K_{\mathrm{u}(\mathrm{eff})}+M_{\mathrm{s}}^{2} /\left(2 \mu_{0}\right)
$$

where $K_{\mathrm{u}(\mathrm{eff})}$ is the effective magnetic anisotropy measured by a torque magnetometer. The $M_{\mathrm{s}}$ values were measured with a vibrating sample magnetometer (VSM). At $t=2.0 \mathrm{~nm}$, the magnetic easy-axis was perpendicular to the film plane, and the magnitude of $K_{\mathrm{u}}$ was about $2.1 \times 10^{6} \mathrm{~J} \cdot \mathrm{m}^{-3}$ with $M_{\mathrm{s}}=1.95 \mathrm{~Wb} \cdot \mathrm{m}^{-2}$. At $t \geq 3.0 \mathrm{~nm}$, the effective magnetic hard-axis was perpendicular to the film plane, and the value of $K_{\mathrm{u}}$ decreased with increasing $t$, reaching $6.8 \times 10^{5} \mathrm{~J} \cdot \mathrm{m}^{-3}$ at $t=20.0 \mathrm{~nm}$. The $K_{\mathrm{u}}$ value obtained at $t \geq 10.0 \mathrm{~nm}$ with $c / a \approx 1.01$ can be attributed to the effect of $B 2$ ordering (in addition to the tetragonal distortion) ${ }^{9}$.

Although the magnetization curves obtained for the samples with $t \geq 3.0 \mathrm{~nm}$ (Fig. 1b) correspond to the effective magnetic hard-axis perpendicular to the film plane, the magnetic easy-axis can be oriented perpendicularly to the film plane after correcting for the demagnetizing field characterized by the $\mu_{0} H_{\mathrm{d}}$ value (equation (3)) of around 2.0 T. Figure $1 \mathrm{~d}$ shows the $t$ dependence of the product $K_{\mathrm{u}} \times t$. The intersection of the linear extrapolation 
a

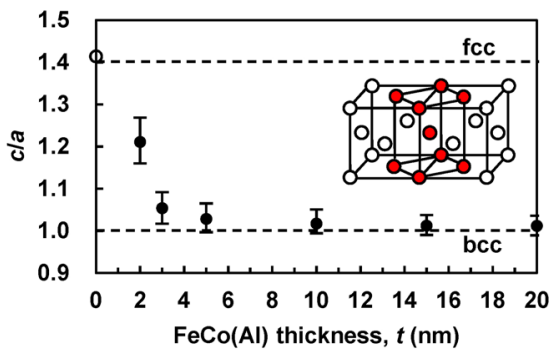

b

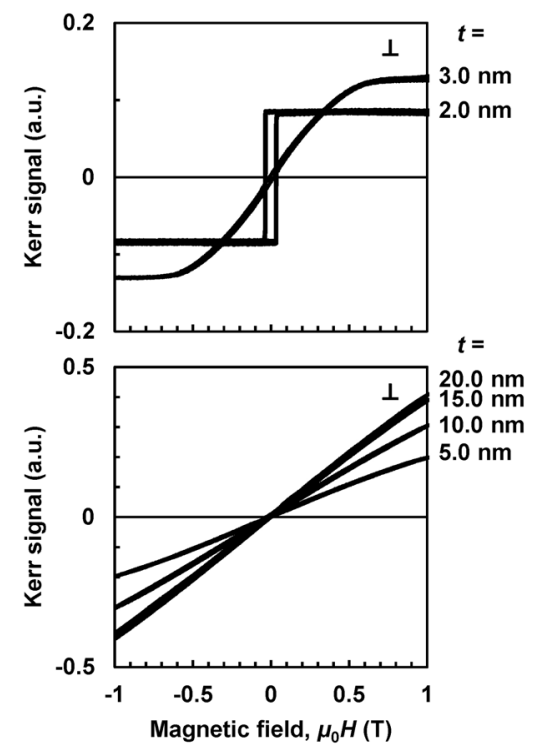

C
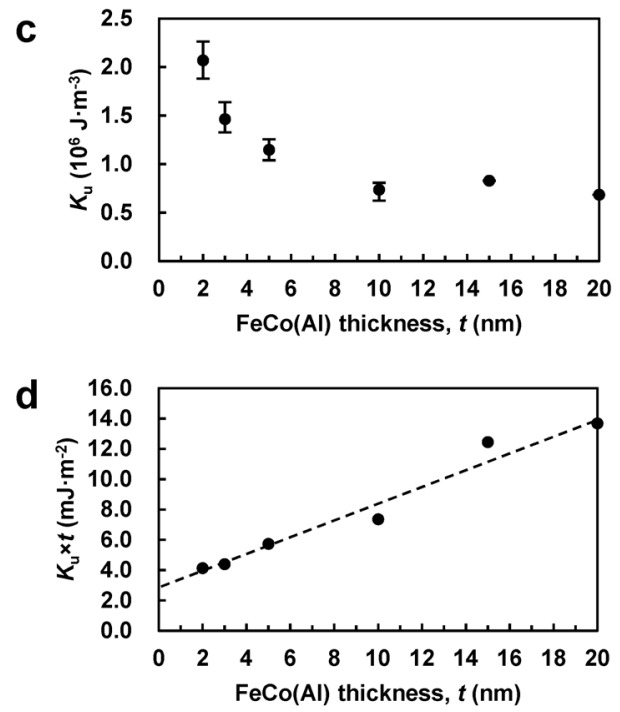

Figure 1. Crystal structure and magnetic properties of the continuous $\mathrm{FeCo}(\mathrm{Al})$ thin films. (a) $t$ dependence of $c / a$. The error bars were calculated from the full widths at half maximum of the XRD peaks. Inset: illustration of the relationship between the fcc and bct unit cells at $c / a=1.41$. The broken lines plotted at $c / a=1.00$ and 1.41 correspond to the bcc and fcc structures, respectively. (b) Perpendicular magnetization curves $(\perp)$. (c) $t$ dependence of $K_{\mathrm{u}}$. The error bars were calculated from the noise widths of the magnetization curves recorded by the VSM. (d) $t$ dependence of $K_{\mathrm{u}} \times t$.

of the obtained data with the vertical axis corresponds to the interfacial anisotropy $\left(K_{\mathrm{i}}\right)^{28}$, yielding a value of around $2.8 \mathrm{~mJ} \cdot \mathrm{m}^{-2}$. Such a small value indicates that $K_{\mathrm{i}}$ is negligible in this system (in other words, the magnitude of $K_{\mathrm{u}}$ was entirely related to magnetocrystalline anisotropy). Recent first-principles calculations also indicated 


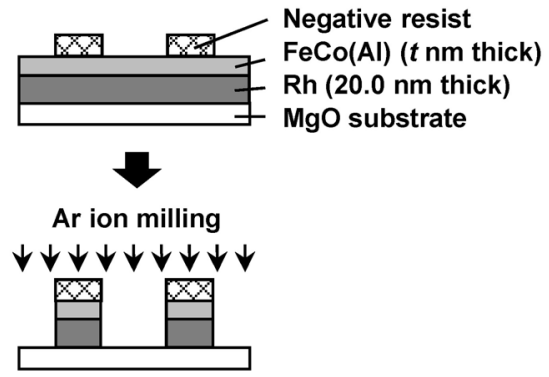

b

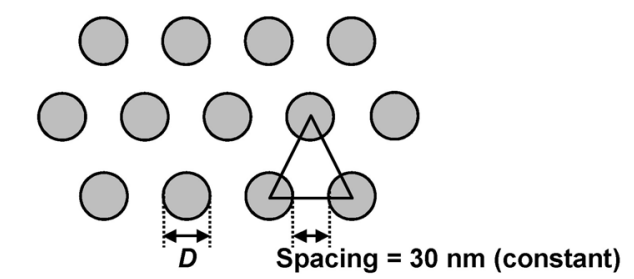

Figure 2. Nanopatterning process. (a) Fabrication via electron beam lithography. (b) A top view of the obtained dot patterns.

that the $K_{\mathrm{i}}$ estimated for the $\mathrm{FeCo} / \mathrm{Rh}$ interface was relatively small ${ }^{11}$ and attributed the origin of the observed uniaxial magnetic anisotropy to the hybridization of the $d_{x y}$ and $d_{x 2-y 2}$ states of bct FeCo via spin-orbit interactions ${ }^{9,10,22}$. The [001] orientation of the bct $\mathrm{FeCo}(\mathrm{Al})$ films was perpendicular to the film plane; hence, uniaxial magnetocrystalline anisotropy was observed along the perpendicular direction due to spin-orbit interactions. The obtained data contradicts the results of previous studies, where the experimental $K_{\mathrm{u}} \times t-t$ plot constructed for the $\mathrm{CoFeB} / \mathrm{MgO}$ system exhibited a negative slope, indicating that the uniaxial magnetic anisotropy existed only at the $\mathrm{CoFeB} / \mathrm{MgO}$ interface. Therefore, it can be concluded that the observed anisotropy was due to the hybridization between the CoFeB Fe-3d and MgO O- $2 p$ orbitals ${ }^{29}$.

Conversion from soft to hard magnetic material by nanopatterning. In order to evaluate the hard magnetic properties of $\mathrm{FeCo}(\mathrm{Al})$, circular dot patterns were fabricated using electron beam lithography and $\mathrm{Ar}$ ion milling techniques (Fig. 2a). Figure $2 \mathrm{~b}$ shows the top view of the resulting dot pattern, where the diameter of the produced circular dots is denoted as $D$. The inter-dot spacing was $30 \mathrm{~nm}$ for all samples.

Figure $3 \mathrm{a}-\mathrm{f}$ show the scanning electron microscopy (SEM) and demagnetized magnetic force microscopy (MFM) images of the patterned samples with $t=2.0 \mathrm{~nm}$ and $D=100,50$, and $30 \mathrm{~nm}$, respectively. Before the MFM measurements, the samples were demagnetized by applying the in-plane magnetic field with a $\mu_{0} H$ of 1.8 T. The dots characterized by a multidomain structure, in which a bright core was surrounded by a dark ring, were observed at $D=100$ and $50 \mathrm{~nm}$ (Fig. $3 \mathrm{~d}$ and e). In contrast, dots with either a bright or dark contrast were observed at $D=30 \mathrm{~nm}$ (Fig. 3f), indicating that the single domain diameter in the remanent state was around $30 \mathrm{~nm}$ at $t=2.0 \mathrm{~nm}$.

Figure 4a shows the normalized perpendicular magnetization curves of the patterned samples recorded using the MOKE technique. The magnetic easy-axis of all the samples was perpendicular to the film plane; even the effective magnetic hard-axis was perpendicular to the plane of the continuous films with $t \geq 3.0 \mathrm{~nm}$ (Fig. 1b). The $N_{\mathrm{t}}$ values (equations (4) and (5)) obtained for the continuous films and dots with $t=20.0 \mathrm{~nm}$ and $D=50 \mathrm{~nm}$ (denoted by the triangles) were approximately 1.0 and 0.59 , respectively. The reduction in $N_{\mathrm{t}}$ decreased $\mu_{0} H_{\mathrm{d}}$ by $40 \%$ from $2.0 \mathrm{~T}$ to $1.2 \mathrm{~T}$; hence, the magnetic easy-axis orientation was changed from the in-plane one to that perpendicular to the film plane because of the change in the aspect ratio $D / t$.

Figure $4 \mathrm{~b}$ shows the $D$ dependence of $\mu_{0} H_{c}$, which includes both the experimental results (the filled symbols) and data calculated via Landau-Lifschitz-Gilbert (LLG) micromagnetic simulations (the open symbols). The $K_{\mathrm{u}}$ values representing structural relaxation, which were plotted as functions of $t$ (Fig. 1c) were used as the LLG parameters. The experimental $\mu_{0} H_{\mathrm{c}}$ value obtained for the continuous film with $t=2.0 \mathrm{~nm}$ was equal to $0.025 \mathrm{~T}$; its magnitude increased to $0.08,0.23$, and $0.36 \mathrm{~T}$ for the patterned samples with $D=100,50$, and $30 \mathrm{~nm}$, respectively. The negative correlation between $\mu_{0} H_{\mathrm{c}}$ and $D$ was confirmed by conducting LLG simulations. Although a single domain was experimentally and theoretically observed for the dot with $t=2.0 \mathrm{~nm}$ and $D=30 \mathrm{~nm}$ (Fig. 3f), its experimental coercivity $(0.36 \mathrm{~T})$ did not reach the calculated $\mu_{0} H_{c}$ value of $1.4 \mathrm{~T}$ (not shown), indicating the existence of a high demagnetizing field and formation of nucleation sites represented by factor $p$ (equation (6)).

The experimental $\mu_{0} H_{c}$ value obtained for the samples with $D=50 \mathrm{~nm}$ almost tripled after increasing $t$ from 2.0 to $20.0 \mathrm{~nm}$, which was likely due to the reduction of the demagnetizing field applied to the nucleation sites, whose $\mu_{0} H_{\mathrm{d}}$ value decreased by $37 \%$ after increasing $t$ from 2.0 to $20.0 \mathrm{~nm}$ at $D=50 \mathrm{~nm}$. For instance, when $t$ was increased to $50.0 \mathrm{~nm}$ at a constant value of $D$ maintained at $50 \mathrm{~nm}$ (to ensure that $D / t \approx 1.0$ ), the calculated $N_{\mathrm{t}}$ value decreased to approximately 0.33 ; consequently, the magnitude of $\mu_{0} H_{\mathrm{d}}$ exhibited a $65 \%$ decrease with respect to the value obtained at $t=2.0 \mathrm{~nm}$. Thus, the coercivity of $\mathrm{FeCo}(\mathrm{Al})$ increases with an increase in $t$ and/or decrease in $D$ until a single domain undergoes coherent rotation. Moreover, increasing the order parameter (via 


\section{a}

\section{$t=2.0 \mathrm{~nm}$}

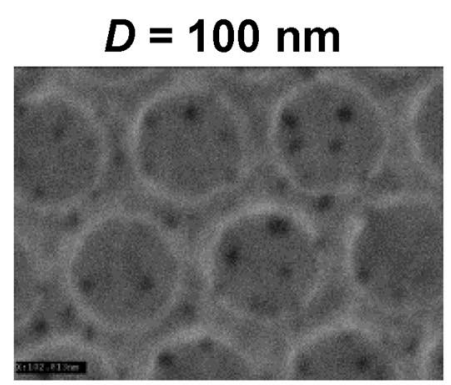

$100 \mathrm{~nm}$

d

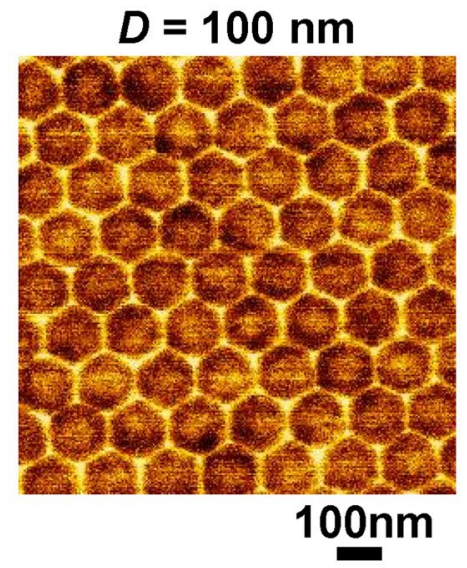

b

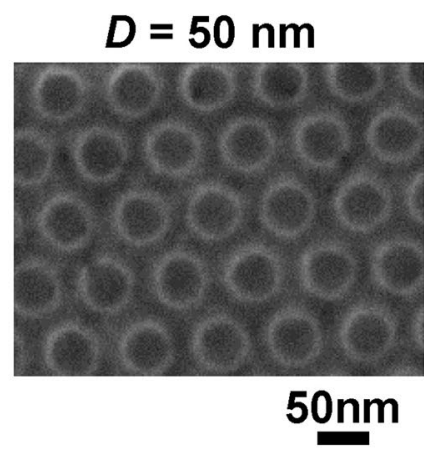

e

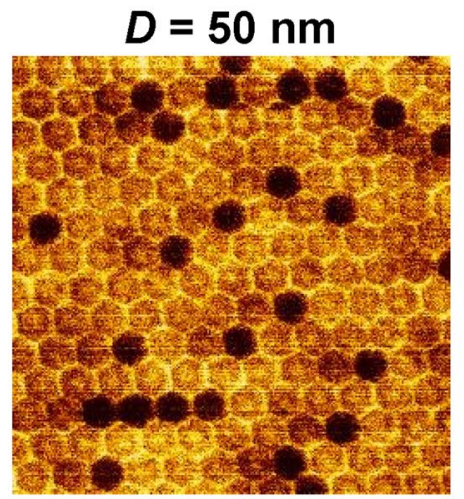

$100 \mathrm{~nm}$
C

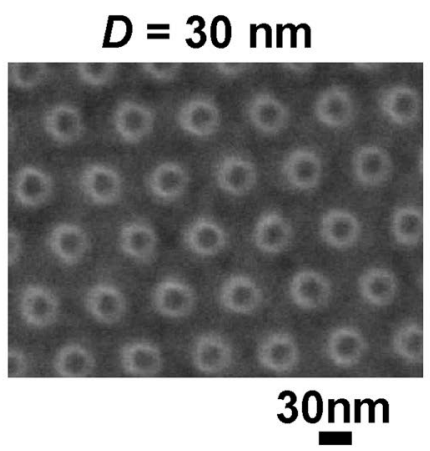

f

$$
D=30 \mathrm{~nm}
$$

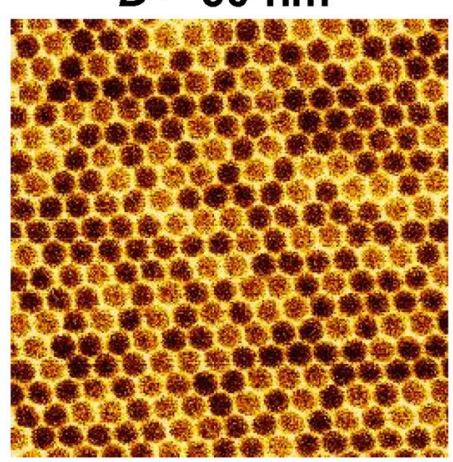

$100 \mathrm{~nm}$

Figure 3. Surface topography and magnetic domains of the fabricated dots. (a-c) SEM images. (d-f) Demagnetized MFM images.

a

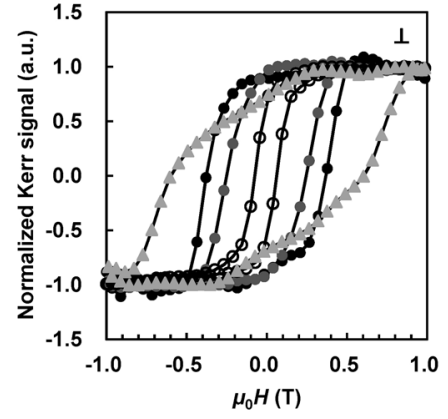

b

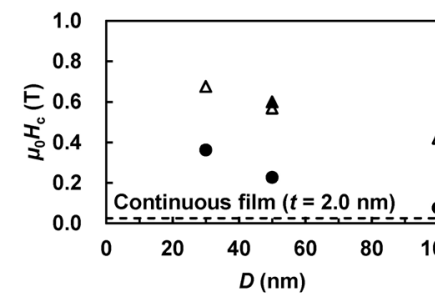

$t=2.0 \mathrm{~nm}$

- $D=30 \mathrm{~nm}$

$D=50 \mathrm{~nm}$

O $D=100 \mathrm{~nm}$

$t=20.0 \mathrm{~nm}$

$\triangle D=50 \mathrm{~nm}$

Figure 4. Magnetic properties of the nanopatterned samples. (a) Normalized perpendicular magnetization curves $(\perp)$. (b) $D$ dependence of $\mu_{0} H_{\mathrm{c}}$ determined via experimental measurements (filled symbols) and theoretical simulations (open symbols). 
annealing) to reduce the number of nucleation sites and thus increase the magnitude of factor $p$ (equation (6)) is also important for reaching high coercivity.

The maximum $\mu_{0} H_{c}$ of $0.60 \mathrm{~T}$ (corresponding to $68 \%$ of the theoretical value calculated using equation (2)) was obtained in this study for the nanopatterned sample with $t=20.0 \mathrm{~nm}$ and $D=50 \mathrm{~nm}$. The experimental $(B H)_{\max }$ value of the nanopatterned sample calculated from the magnetization curve (depicted by the triangles in Fig. $4 \mathrm{a}$ ) and $M_{\mathrm{s}}=1.95 \mathrm{~Wb} \cdot \mathrm{m}^{-2}$ measured by the VSM is $150 \mathrm{~kJ} \cdot \mathrm{m}^{-3}$. Assuming that the single-domain dots exhibit coherent rotation with $M_{\mathrm{s}}=1.95 \mathrm{~Wb} \cdot \mathrm{m}^{-2}$ and the total dot area on the two-dimensional surface equal to $90 \%$, the ideal $(B H)_{\max }$ of this system calculated via equation (1) is expected to be around $687 \mathrm{~kJ} \cdot \mathrm{m}^{-3}$ or $140 \%$ of the value obtained for FeNdB.

\section{Conclusion}

In summary, the tetragonally distorted $\mathrm{FeCo}(\mathrm{Al})$ thin film with a lattice parameter ratio $c / a$ of $1.01-1.21$ and high $K_{\mathrm{u}}$ of $2.1 \times 10^{6} \mathrm{~J} \cdot \mathrm{m}^{-3}$ was fabricated. Further, a coercivity of $0.60 \mathrm{~T}$ was obtained for the nanopatterned FeCo layer with a thickness of $20.0 \mathrm{~nm}$ and dot pattern diameter of $50 \mathrm{~nm}$. These results demonstrate that the combination of lattice engineering (introduction of tetragonal distortion into the structure of FeCo alloys) and nanopatterning (fabrication of patterns smaller than the single domain size) yields materials possessing high values of $K_{\mathrm{u}}, H_{\mathrm{c}}$, and $M_{\mathrm{s}}$. Therefore, the produced materials exhibit high potentials for future applications requiring high magnetic anisotropy and flux density, such as high-performance permanent magnets for motors and data storage devices.

\section{Methods}

$\mathrm{FeCo}(\mathrm{Al})(t=2.0-20.0 \mathrm{~nm})$ films were prepared via dc-magnetron co-sputtering at a base pressure of $10^{-7} \mathrm{~Pa}$ and Ar gas pressure of $0.1 \mathrm{~Pa}$. The composition of the produced films was controlled by varying the sputtering rates of the utilized $\mathrm{Fe}, \mathrm{Co}$, and $\mathrm{Al}$ targets and was determined using an electron probe $\mathrm{X}$-ray microanalyser with an error of less than 1 at.\% by averaging the compositions of 10 points on the surfaces of the film samples with dimensions of $1 \mathrm{~cm} \times 1 \mathrm{~cm}$. First, a $20.0 \mathrm{~nm}$ thick Rh buffer layer was grown on a single-crystalline $\mathrm{MgO}$ (100) substrate at $673 \mathrm{~K}$. After decreasing the temperature to $473 \mathrm{~K}, \mathrm{FeCo}(\mathrm{Al})$ films were prepared. Finally, a $2.0 \mathrm{~nm}$ thick $\mathrm{SiO}_{2}$ capping layer was sputtered onto the $\mathrm{FeCo}(\mathrm{Al})$ surface at $298 \mathrm{~K}$. Calixarene-type resist (TEBN-1, Tokuyama Corp., Japan) was used during nanopatterning via electron beam lithography. The total area of the dot pattern was about $120 \mu \mathrm{m} \times 120 \mu \mathrm{m}$ for each sample. The crystalline structure of the produced films was investigated via the out-of-plane and in-plane XRD with $\mathrm{CuK} \alpha$ radiation. The degree of order parameter was measured by XRD with synchrotron radiation at a photon energy of $7.1 \mathrm{keV}$. The magnetization curves were recorded using the MOKE method under a magnetic field of up to $1.0 \mathrm{~T}$ applied perpendicularly to the film plane. The laser diameter of the MOKE measurement system was about $100 \mu \mathrm{m}$ and significantly smaller than the patterned area of $120 \mu \mathrm{m} \times 120 \mu \mathrm{m}$. The $M_{\mathrm{s}}$ values were measured by the VSM with a magnetic field of up to $2.2 \mathrm{~T}$. $K_{\mathrm{u}(\text { eff) }}$ was measured using a torque magnetometer with a magnetic field of up to $2.5 \mathrm{~T}$. The sample surface topography and related magnetic domains were observed in vacuum by SEM and MFM, respectively. The cell size for the LLG simulations was $2 \mathrm{~nm} \times 2 \mathrm{~nm} \times 2 \mathrm{~nm}$. The $t$-dependent $K_{\mathrm{u}}$ values $\left(K_{\mathrm{u}}=2.1 \times 10^{6}, 1.5 \times 10^{6}, 1.1 \times 10^{6}\right.$, and $6.8 \times 10^{5} \mathrm{~J} \cdot \mathrm{m}^{-3}$ for the regions with $0 \leq t<2.0 \mathrm{~nm}, 2.0 \leq t<4.0 \mathrm{~nm}, 4.0 \leq t<8.0 \mathrm{~nm}$, and $8.0 \leq t \leq 20.0 \mathrm{~nm}$, respectively) as well as $M_{\mathrm{s}}=1.95 \mathrm{~Wb} \cdot \mathrm{m}^{-2}$ and the exchange stiffness constant of $1.8 \times 10^{-11} \mathrm{~J} \cdot \mathrm{m}^{-1}$ were used as the LLG parameters.

\section{References}

1. Skomski, R. et al. Predicting the future of permanent-magnet materials. IEEE Trans. Magn. 49, 3215-3220, https://doi.org/10.1109/ TMAG.2013.2248139(2013).

2. Terris, B. D. \& Thomson, T. Nanofabricated and self-assembled magnetic structures as data storage media. J. Phys. D: Appl. Phys. 38, R199-R222, https://doi.org/10.1088/0022-3727/38/12/R01 (2005).

3. Hirohata, A. et al. Roadmap for emerging materials for spintronic device applications. IEEE Trans. Magn. 51, 1-11, https://doi. org/10.1109/TMAG.2015.2457393 (2015).

4. Coey, J. M. D. Magnetism and Magnetic Materials, 10-39 (Cambridge University Press, New York, (2009).

5. Wohlfarth, E. P. Ferromagnetic Materials Vol. 2, 168-188 (North-Holland Publishing Company, Amsterdam, (1980).

6. Sundar, R. S. \& Deevi, S. C. Soft magnetic FeCo alloys: alloy development, processing, and properties. Int. Mater. Rev. 50, 157-192, https://doi.org/10.1179/174328005X14339 (2005).

7. Burkert, T., Nordsröm, L., Eriksson, O. \& Heinonen, O. Giant magnetic anisotropy in tetragonal FeCo alloys. Phys. Rev. Lett. 93, 027203, https://doi.org/10.1103/PhysRevLett.93.027203 (2004)

8. Turek, I., Kudrnovský, J. \& Carva, K. Magnetic anisotropy energy of disordered tetragonal Fe-Co systems from ab initio alloy theory. Phys. Rev. B 86, 174430, https://doi.org/10.1103/PhysRevB.86.174430 (2012).

9. Kota, Y. \& Sakuma, A. Degree of order dependence on magnetocrystalline anisotropy in body-centered tetragonal FeCo alloys. Appl. Phys. Express 5, 113002, https://doi.org/10.1143/APEX.5.113002 (2012).

10. Kota, Y. \& Sakuma, A. Mechanism of uniaxial magnetocrystalline anisotropy in transition metal alloys. J. Phys. Soc. Japan 83, 034715, https://doi.org/10.7566/JPSJ.83.034715 (2014).

11. Hyodo, K., Kota, Y. \& Sakuma, A. Theoretical evaluation of perpendicular magnetic anisotropy of bct-Fe $\mathrm{Fe}_{50} \mathrm{Co}_{50}$ stacked on Rh. J. Magn. Soc. Japn 39, 37, https://doi.org/10.3379/msjmag.1503R002 (2015).

12. Andersson, G. et al. Perpendicular magnetocrystalline anisotropy in tetragonally distorted Fe-Co alloys. Phys. Rev. Lett. 96, 037205, https://doi.org/10.1103/PhysRevLett.96.037205 (2006).

13. Winkelmann, A., Przybylski, M., Luo, F., Shi, Y. \& Barthel, J. Perpendicular magnetic anisotropy induced by tetragonal distortion of FeCo alloy films grown on Pd(001). Phys. Rev. Lett. 96, 257205, https://doi.org/10.1103/PhysRevLett.96.257205 (2006).

14. Luo, F., Fu, X. L., Winkelmann, A. \& Przybylski, M. Tuning the perpendicular magnetic anisotropy in tetragonally distorted Fe $\mathrm{Co}_{1-\mathrm{x}}$ alloy films on Rh (001) by varying the alloy composition. Appl. Phys. Lett. 91, 262512, https://doi.org/10.1063/1.2821370 (2007).

15. Yildiz, F., Przybylski, M., Ma, X.-D. \& Kirschner, J. Strong perpendicular anisotropy in $\mathrm{Fe}_{1-\mathrm{x}} \mathrm{Co}_{\mathrm{x}}$ alloy films epitaxially grown on mismatching Pd(001), Ir(001), and Rh(001) substrates. Phys. Rev. B 80, 064415, https://doi.org/10.1103/PhysRevB.80.064415 (2009).

16. Reichel, L. et al. Increased magnetocrystalline anisotropy in epitaxial Fe-Co-C thin films with spontaneous strain. J. Appl. Phys. 116, 213901, https://doi.org/10.1063/1.4901595 (2014).

17. Ohtsuki, T. et al. Magnetic domain observation of FeCo thin films fabricated by alternate monoatomic layer deposition. J. Appl. Phys. 115, 043908, https://doi.org/10.1063/1.4862910 (2014). 
18. Wang, B., Oomiya, H., Arakawa, A., Hasegawa, T. \& Ishio, S. Perpendicular magnetic anisotropy and magnetization of L1 ${ }_{0} \mathrm{FePt} /$ FeCo bilayer films. J. Appl. Phys. 115, 133908, https://doi.org/10.1063/1.4870463 (2014).

19. Wang, B. et al. Investigation of magnetic anisotropy and magnetic moments of tetragonal distorted $\mathrm{Fe}_{1-x} \mathrm{Co}_{\mathrm{x}}$ films on $\mathrm{L} 1_{0} \mathrm{FePt}$ underlayer. J. Appl. Phys. 117, 17C709, https://doi.org/10.1063/1.4907691 (2015).

20. Lao, B., Jung, J. W. \& Sahashi, M. Strong perpendicular uniaxial magnetic anisotropy in tetragonal $\mathrm{Fe}_{0.5} \mathrm{Co}_{0.5}$ films of artificially ordered B2 state. IEEE Trans. Magn. 50, 2008704, https://doi.org/10.1109/TMAG.2014.2322936 (2014).

21. Matsuura, M., Tezuka, N. \& Sugimoto, S. Increased uniaxial perpendicular anisotropy in tetragonally distorted FeCo-Ti-N films. J. Appl. Phys. 117, 17A738, https://doi.org/10.1063/1.4916763 (2015).

22. Oomiya, H. et al. Tetragonally distorted structure and uniaxial magnetic anisotropy of $\mathrm{Fe}_{100-x} \mathrm{Co}_{\mathrm{x}} / \mathrm{Rh} / \mathrm{MgO}$ epitaxial films. J. Phys. D: Appl. Phys. 48, 475003, https://doi.org/10.1088/0022-3727/48/47/475003 (2015).

23. Thomson, T., Hu, G. \& Terris, B. D. Intrinsic distribution of magnetic anisotropy in thin films probed by patterned nanostructures. Phys. Rev. Lett. 96, 257204, https://doi.org/10.1103/PhysRevLett.96.257204 (2006).

24. Bublat, T. \& Goll, D. Influence of dot size and annealing on the magnetic properties of large-area $\mathrm{L} 1_{0}$-FePt nanopatterns. J. Appl. Phys. 110, 073908, https://doi.org/10.1063/1.3646550 (2011).

25. Hasegawa, T., Kasahara, R., Sasaki, K. \& Ishio, S. Microfabrication of FeMnPt films involving magnetic phase change due to structural transformation caused by ion irradiation. Phys. Status Solidi RRL 10, 498-502, https://doi.org/10.1002/pssr.201600031 (2016).

26. Raynor, G. V. \& Rivlin, V. G. Phase Equilibria in Iron Ternary Alloys: A Critical Assessment of the Experimental Literature, 71-81 (Institute of Metals, London, (1988).

27. Yoshida, S. et al. Uniaxial magnetic anisotropy of tetragonal FeCo and FeCoAl films. The 8th Joint European Magnetic Symposia, p. $757, \mathrm{UK}(2016)$

28. Johnson, M. T., Bloemen, P. J. H., den Broeder, F. J. A. \& de Vries, J. J. Magnetic anisotropy in metallic multilayers. Rep. Prog. Phys. 59, 1409-1458, https://doi.org/10.1088/0034-4885/59/11/002 (1996).

29. Ikeda, S. et al. A perpendicular-anisotropy CoFeB-MgO magnetic tunnel junction. Nat. Mater. 9, 721-724, https://doi.org/10.1038/ nmat2804 (2010).

\section{Acknowledgements}

We thank A. Arakawa and S. Yoshida for technical support and the assistance with sample preparation. We also thank Dr. N. Inami, Dr. T. Ueno, and Dr. K. Ono for their help with conducting XRD measurements using synchrotron radiation, and Prof. S. Saito for the assistance with the torque measurements. This work was supported by the Japan Society for the Promotion of Science KAKENHI through its Grant-in-Aid for Young Scientists (A) program (JP15H05518), and through a program of the New Energy and Industrial Technology Development Organization (NEDO), Japan. We would like to thank Editage and Dr. E. Watters for English language editing.

\section{Author Contributions}

T.H. and S.I. supervised the study. T.H. wrote the manuscript. K.T., K.K., and M.H. prepared the samples. S.K. measured magnetic properties. M.K. observed the magnetic domains. All authors discussed the results and commented on the manuscript.

\section{Additional Information}

Competing Interests: The authors declare that they have no competing interests.

Publisher's note: Springer Nature remains neutral with regard to jurisdictional claims in published maps and institutional affiliations.

Open Access This article is licensed under a Creative Commons Attribution 4.0 International License, which permits use, sharing, adaptation, distribution and reproduction in any medium or format, as long as you give appropriate credit to the original author(s) and the source, provide a link to the Creative Commons license, and indicate if changes were made. The images or other third party material in this article are included in the article's Creative Commons license, unless indicated otherwise in a credit line to the material. If material is not included in the article's Creative Commons license and your intended use is not permitted by statutory regulation or exceeds the permitted use, you will need to obtain permission directly from the copyright holder. To view a copy of this license, visit http://creativecommons.org/licenses/by/4.0/.

(C) The Author(s) 2017 